\title{
Tensor Lines in Tensor Fields of Arbitrary Order
}

\author{
Mario Hlawitschka ${ }^{1}$ and Gerik Scheuermann ${ }^{1}$ and Alfred Anwander ${ }^{2}$ and \\ Thomas Knösche ${ }^{2}$ and Marc Tittgemeyer ${ }^{3}$ and Bernd Hamann ${ }^{4}$ \\ University of Leipzig, Germany ${ }^{1}$ \\ Max Planck Institute for Human Cognitive and Brain Science, Leipzig, Germany ${ }^{2}$ \\ Max Planck Institute for Neurological Research, Cologne, Germany ${ }^{3}$ \\ Institute for Data Analysis and Visualization (IDAV) and \\ Department of Computer Science, University of California, Davis, USA ${ }^{4}$
}

\begin{abstract}
This paper presents a method to reduce time complexity of the computation of higher-order tensor lines. The method can be applied to higher-order tensors and the spherical harmonics representation, both widely used in medical imaging. It is based on a gradient descend technique and integrates well into fiber tracking algorithms. Furthermore, the method improves the angular resolution in contrast to discrete sampling methods which is especially important to tractography, since there, small errors accumulate fast and make the result unusable. Our implementation does not interpolate derived directions but works directly on the interpolated tensor information. The specific contribution of this paper is a fast algorithm for tracking lines tensor fields of arbitrary order that increases angular resolution compared to previous approaches.
\end{abstract}

\section{Introduction}

Tensor data have a long history in engineering. The studies of Basser et al. [1, $2]$ introduced the diffusion tensor to medical imaging of the human brain. In human brain imaging applications, it is now possible to measure anisotropic diffusion of hydrogen that relates to the major neural fiber bundles in vivo. More recent methods focus on improving the diffusion model by using the higher angular resolution of scanners that are now available even in clinical environment due to the sustaining decrease in scanning times. While second-order diffusion tensor imaging is only meaningful in isotropic areas or in areas where a voxel contains a single fiber bundle, many voxels in human brain scans contain multiple fiber bundles [3]. To overcome these well-known limitations, multi-tensor models have been proposed that match the sum of more than one tensor in every voxel, where usually the number of fibers has to be known in advance. In contrast to this, other methods, including Frank's high angular resolution diffusion imaging (HARDI) [4,5], Tuch's q-ball imaging [6,7], Alexander's PASMRI [8] and higher-order tensor approaches by Özarslan et al. [9] do not base on apriori knowledge. They all derive a spherical function that is used to estimate the direction of fibers. Depending of the approach, this function is described by higher-order tensors, spherical harmonics or discrete, spherical sample points. 
Fiber tracking has become popular in recent years, and many groups started implementing fiber tracking for second-order tensors $[10,11]$ or higher-order methods [3]. Most of these methods split computation of directions from the tracking itself which leads to problems in areas, where the direction changes rapidly. The only method that is in some way comparable to our approach has been presented by Weinstein et al. [12]. Their method uses an advection-diffusion progress to propagate lines through second-order tensor fields. While their major intention is to use the advection as a smoothing process to provide tracking in areas of isotropic diffusion or noisy data, our method may be interpreted by its function - but not its physical meaning - as the advection step, but uses this step to compute the exact directions that can not be found analytically. The result is a line that is tangential to the directions at every position sampled by the line which is true for Weinstein's method only in constant tensor fields.

We use methods of Özarslan and Frank and describe the function as higherorder tensors and spherical harmonics. Both representations describe the same function space and can be used interchangeable as a basis set for our algorithm. While their method sets the basic idea for the storage of the local data, we show, how directions of fibers can be found efficiently and consistent tracking is performed using the higher-order information in every tracking step. Furthermore, all smooth, scalar, square integrable functions on the sphere can be approximated by this basis set, therefore, it can be easily adapted to methods such as PASMRI where the "persistent angular structure" has similar function like the orientation distribution function (ODF) computed in q-ball imaging that we use. While the method demonstrated to work on data acquired using medical imaging, the underlying theory is independent of the application and the tracking can be extended to other fields of science, e.g., mechanical engineering, where higher-order tensors are used to describe material properties.

In the next section we present the basics of higher-order tensor lines previously presented by Hlawitschka et al. [13] that are needed to understand this paper. Then we improve the previously presented method using gradient descend methods to make them computationally efficient. The resulting algorithm is capable of painting lines in about the same speed as second-order tensor methods and have a much higher angular precision than grid based methods. The increased local accuracy improves fiber tracking tremendously as drawing integral lines is highly sensitive to small local errors.

\section{$2 \quad$ Higher-Order Tensor Lines}

Higher-order tensor lines [13] are a generalization of streamlines and secondorder tensor lines to tensors of arbitrary order. They have been defined as lines, following the maxima, minima or saddle points on the scalar surface function describing local properties. The function can be defined, for example, by the tensor $T$ and a direction $\boldsymbol{g}$ on the unit sphere $S^{2}$ as parameter by

$$
f_{s}(\boldsymbol{g})=T_{i_{1} \ldots i_{n}} \boldsymbol{g}_{i_{1}} \ldots \boldsymbol{g}_{i_{n}} .
$$


If $T$ is a second-order tensor, i.e., $n=2$ this is the standard case found in mechanical engineering, e.g., as derivative of vector fields as well as in secondorder diffusion tensor imaging. Higher order tensors are mainly used in medical imaging which is the major motivation for this formulation. The function $f_{s}$ can be represented by a spherical height-field as used later in Fig. 3, resulting in a surface that can be used as a glyph representation. For simplicity, we call the field tensor field, even though it is not limited to the tensor formulation provided in Eq. 1, but $f_{s}(\boldsymbol{g})$ may be written in spherical harmonics basis, too, eventually leading to the same formulation of lines.

Let $\mathbf{T}$ be a tensor field defined on $D \subseteq \mathbb{R}^{3}$. If the field is not degenerate there exist open, connected sets $U_{m} \subseteq D$ and functions $\hat{g}_{m}(x), x \in U_{m}$ where $\hat{h}(x)$ is a vector valued function indicating the direction of the $m$-th local maximum of $f_{s}$. We already have shown in [13] that the function can be chosen that $\hat{g}(x)$ is $C^{1}$ continuous on $U_{m}$. The function $\hat{h}$ can be interpreted as a vector field on $U_{m}$. Then a major tensor line through the point $x_{0}$ on the field defined by $\hat{h}(x)$ is the curve $c(t)$ where

$$
c(0)=x_{0} \quad \text { and } \quad \frac{\partial c(t)}{\partial t}=\hat{h}(x) \quad \text { for } \quad t \geq 0
$$

It is important to see that as there may exist many local maxima $m$ at any position $x$ there may exist a large number of different, $C^{1}$-continuous vector fields $U_{m_{1}} \ldots U_{m_{n}}$ containing the same point $x$ which makes many different lines go through the same point $x$. As the structure of these neighborhoods is quite complex and there currently is no method to compute them analytically, it is not possible to extract the vector fields first, followed by painting streamlines in these vector fields. Although computing multiple vectors locally and finding the best matching direction does not provide good results because direction information between sample points has to be interpolated. Obviously, before interpolating the data, it has to be guaranteed that all vectors interpolated belong to the same locally defined vector field, which cannot be computed by only taking information of the local directions into account. Instead, it is possible to use the property of $C^{1}$ continuity of these vector fields to fill this gap. The problem of finding the locally defined field $\hat{h}$ efficiently is addressed first. Then we present an algorithm using the continuity property of the field to extract higher-order tensor lines by implicitly constructing the local vector field.

\subsection{Search for Local Directions}

All algorithms described in literature base on evaluation of the tensor function on a discrete set of points and, in some cases after applying a sharpening transform, search for local maxima based on these sample points. The result is the same when the grid is seen as a linearly interpolated field. Up to now, this method is state of the art in computer graphics and medical visualization and has been used in many publications, among them Descoteaux et al. [14].

Although this method leads to good results and, given a reasonable amount of sample points, the directions derived are good enough for most cases, we 

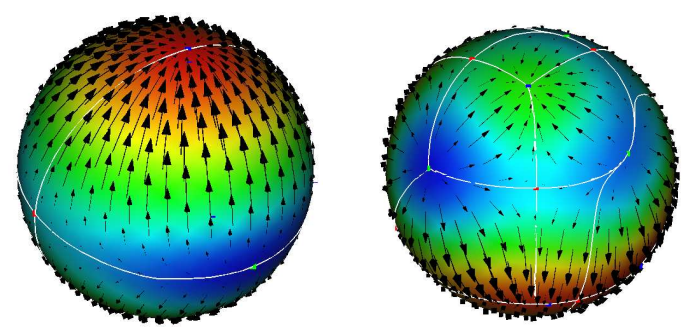

Fig. 1. Simple glyphs out of a data set. Black arrows indicate the gradient field. Vector field topology is indicated with sources (green) saddles (red) and sinks (blue). The separatrices (white lines) segment the left surface in four parts of similar behavior. A pseudo color-map indicates the underlying scalar function from deep blue (lowest value) via green to red (highest value). Left: second-order tensor. Right: more complex fourth-order tensor based on the same data set.

improve the directions using the underlying interpolation of the tensor model. Assuming, we already have a good starting point that is close to the current fiber direction, Euler's algorithm provides a fast way of finding this maximum. Because the number of steps is small and the field is well conditioned, Euler's approach provides good results. Higher-order approaches such as Runge Kutta integration would lead to more evaluations of the gradient function for small amount of steps. If the angles become larger, this is clearly a tradeoff in precision versus speed, but as the gradient field is a smooth potential vector field, this simple approach already provides good results. As the algorithm depends on the derivative of the scalar field, vector field topology [15] of the derivative of the scalar field can be used to visualize the areas of influence of every maximum as it is shown in Fig. 1.

\subsection{The Algorithm}

We start as all previous methods by sampling the function on a predefined grid and looking for local maxima on this grid. Instead of repeating the search at every position, the following integration algorithm is used:

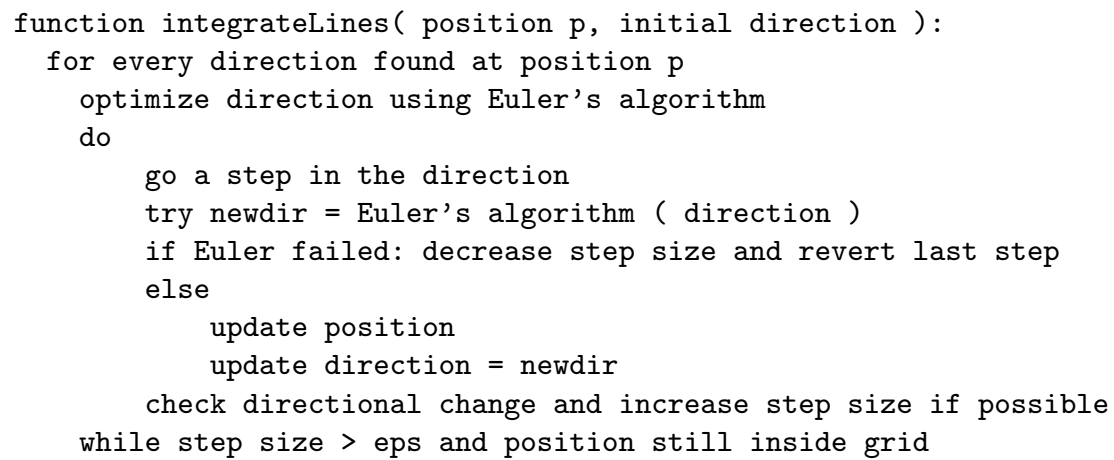


In contrast to most algorithms where the directions are pre-computed and directions are interpolated, our algorithm computes the directions on-the-fly. Therefore, we are able to use the feedback between finding the directions and the line integrator to change the step size of the integrator as shown in Fig. 2.2. A smaller step size of the integrator ensures that the inner Euler algorithm finds the maximum in less time but on the other hand increases the number of steps of the integrator. In our tests, we found that this parameter is not critical for the algorithm, but we kept the step size small to ensure that we find the next direction in at most ten steps. Using adaptive step size further reduced the number of steps in the inner loop and finally improved the precision of the direction. Because of the smoothness of the glyphs, the maximal step size can easily be adapted in a way that no significant maximum is missed and the algorithm always converges.

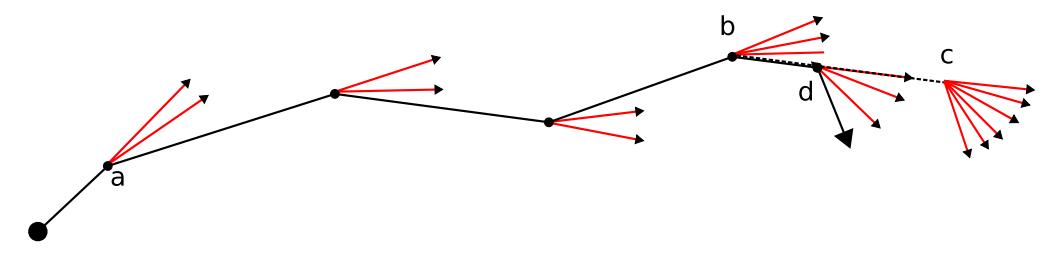

Fig. 2. Illustration of the iteration process using Euler's integration with adaptive step size. The black line shows the actual integration path. Red arrows show the iteration on the glyph for finding the new direction. The dashed line depicts a path that is tried but rejected. After the first step in point a, the step size is increased because of the angular criterion. In point b, a step (dotted line to c) is tried, but finding the direction in c failed because too many iterations are required and the angle becomes large. Therefore, the step size is reduced and the new direction is found in point $\mathrm{d}$. The iteration continues with the smaller step size.

\subsection{Gradient Calculation for Tensor Representations}

The described algorithm needs the surface gradient of the scalar function $f_{s}$ that can be calculated analytically. Let $T$ be a symmetric tensor and let $f(g)$ be a tensor-function as defined in Eq. 1. For simplicity, most of the analysis is done on the sphere $\bar{g} \in S^{2} \subset \mathbb{R}^{3},\|\bar{g}\|=1$. The derivatives along the coordinate axes of the scalar function $f$ are

$$
\boldsymbol{v}(g)=\nabla f(g)=T_{i_{1} \ldots i_{\ell}} g_{i_{1}} \cdot \ldots \cdot g_{i_{\ell-1}}
$$

The radial derivatives are given by projection of the derivative on the radial direction $g$

$$
\begin{aligned}
\boldsymbol{v}_{\boldsymbol{r}}(g) & =\frac{\partial}{\partial r} f(g)=\langle\boldsymbol{v}(g), g\rangle \\
& =T_{i_{1}} i_{\ell} g_{i_{1}} \cdot \ldots \cdot g_{i_{\ell-1}} \bar{g}_{i_{\ell}}=\|g\|^{\ell-1} f(\bar{g}) .
\end{aligned}
$$



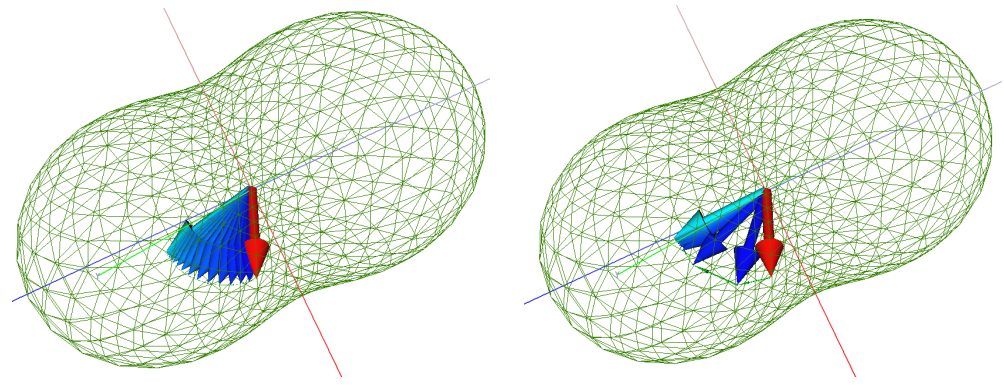

Fig. 3. Gradient descend on the surface function $f_{s}$. The red arrow indicates starting direction of the iteration. The green line indicates the correct new direction which is reached by the cyan vector, too. The green grid shows the scalar function as an offset from the center and is not used in the calculation but only shown to visualize the function. Left: fixed step size, right: Adaptive step size yields faster convergence. The angle between the starting direction and the result is exaggerated here as our algorithm would not allow that large angles.

If $\|g\|=1$ the normalization factor vanishes and the gradient on the surface of the unit sphere can be calculated using

$$
\boldsymbol{v}_{s}=\boldsymbol{v}-\boldsymbol{v}_{\boldsymbol{r}} \cdot \bar{g}=T_{i_{1} \ldots i_{\ell}} \bar{g}_{i_{1}} \cdot \ldots \cdot \bar{g}_{i_{\ell-1}}-T_{j_{1} \ldots j_{\ell}} \bar{g}_{j_{1}} \cdot \ldots \cdot \bar{g}_{j_{\ell}} \bar{g}_{i_{\ell}}
$$

\subsection{Gradient Calculation for Spherical Harmonics}

An alternative representation that can be used to describe the tensor field is the spherical harmonics representation. Here, a finite set of spherical harmonics basis functions [16], i.e., a smooth set of globally defined basis functions on the sphere that can be seen as a spherical analogue to the Fourier basis in 2D is used to represent the measured data. Usually the lowest orders of the spherical harmonics are used to approximate the data, where the order defines the angular resolution. As spherical harmonics are eigenfunctions of the Laplace operator, their derivatives can be represented in the spherical harmonics basis, too and therefore are easy to compute. Using numerical evaluation, one must be aware of instabilities near the z-Axis. The instabilities arise from the unbounded Legendre associated polynomials $P_{\ell}^{1}(\cos (\theta))$. This can be circumvented by rotating the spherical harmonics by $90^{\circ}$ before computing the derivatives. The rotation in spherical harmonics basis representation can be performed using a vector matrix multiplication where the rotation matrix is a sparse block structure matrix as described, e.g., by Ivanic $[17,18]$. Using this approach, derivatives can be computed on spherical harmonics representations of the spherical field with high numerical precision. The matrix to rotate the spherical harmonics depends on the degree of the spherical harmonics and is pre-computed once and is applied to every data point when needed. A $3 \times 3$ rotation matrix is then used to rotate the resulting direction back into the original frame of reference. 


\section{Evaluation and Results}

We applied our algorithm to data acquired with a three Tesla Siemens scanner on a healthy volunteer. 60 diffusion weighted images were acquired using three times averaging and 21 baseline images at $b=2500$. The same gradient information is used to compute a test data set for single fiber distributions. In all cases, the data is prepared using spherical harmonics based q-ball imaging and converted to orientation distribution function using Descoteaux's [19] description of the FunkRadon-Transform for the spherical harmonics basis. The in-slice resolution is $128 \times 128$ voxels and 71 slices are acquired on a $1.7 \times 1.7 \times 1.7 \mathrm{~mm}^{3}$ grid .
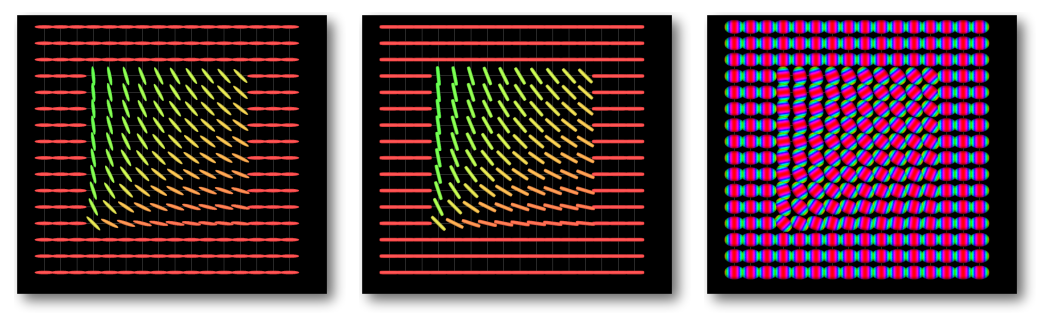

Fig. 4. From left to right: second order ellipsoidal glyphs, second order superquadric glyphs, and fourth-order glyph on q-ball data.

We first test our algorithm for simple test data sets. Fig. 4 shows a simple second-order data set. The data was created from second-order tensors showing a single fiber direction and stored as raw data. For the evaluation the data was reconstructed using linear least-squares fitting to second-order tensors and q-Ball imaging on a spherical harmonics basis of order four. We chose a secondorder model here, because visualization of second-order glyphs can be used to evaluate the precision of our algorithm as there, the behavior of the fiber tracts is well-known. We compared iterative refinement to brute-force search for three different resolutions. While 60 sample points on a regular spherical grid obviously lead to low angular resolution, 240 sample points are still unusable, but even for 960 sample points, our algorithm performs better. As the grid is symmetric, for grid sampling 30, 120 and 480 evaluations of the spherical harmonics are required respectively. Our algorithm usually converges in five steps to an error of approximately 1e-5 in polar angles, therefore, a fixed upper limit of ten steps can be used. In this case, the algorithm would need 50 evaluations of spherical harmonics to compute the maxima, i.e., 30 to compute a rough estimate on the grid and another twenty because computation of the derivative has the same complexity as evaluating the spherical harmonics twice and can be written as a spherical harmonics itself. This means a reduction of evaluations of spherical harmonics from 960 to 50, which improves the overall speed tremendously and still increases the angular resolution. A comparison of the two methods is shown in Fig. 5. 

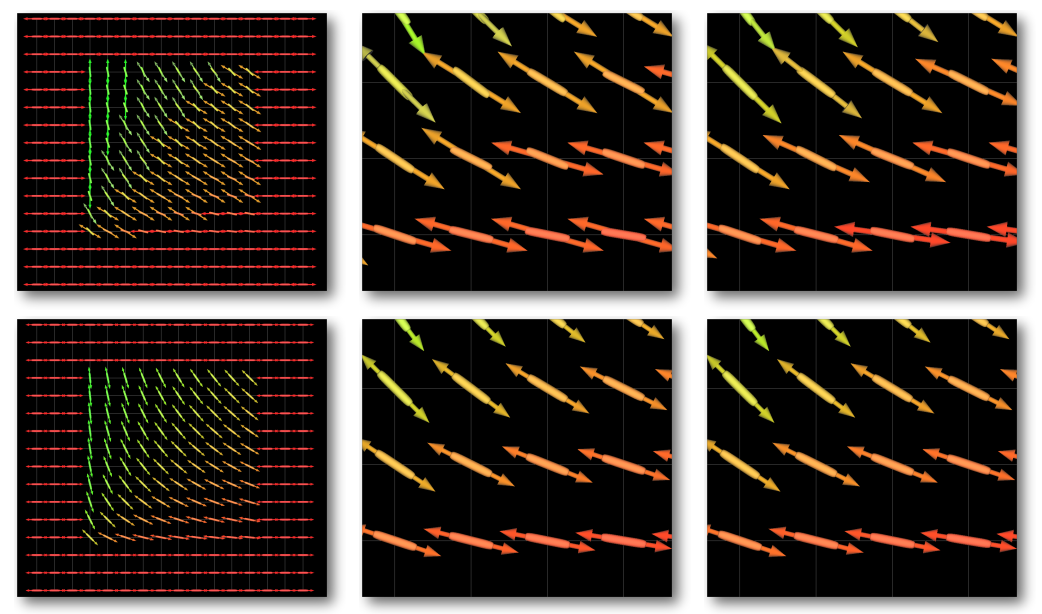

Fig. 5. A comparison of grid based search (top row) and iterative improvement (bottom row) for sampling on a grid containing 60 points (left) and zoomed into the bottom row for 240 points (middle) and 960 points (right). The points were constructed from an icosahedron subdivision. While the arrows indicate the directions found by the fourthorder q-ball imaging, superquadric glyphs are drawn for comparison as they show the true tensor direction. For 240 sample points, the deviation of the naive implementation is clearly visible, but even for 960 points it is visible in the bottom row of arrows. Iterative refinement (bottom row) gives the same results for all three cases, i.e., it is independent of the initial resolution.

On the measured data set of a healthy subject, low FA values make the second-order tensor lines fail while higher-order lines are able to extract the structures (Fig. 6). Our algorithm is able to extract multiple fiber crossings as shown in Fig. 7. There crossings of two fibers can be found, e.g., in the area where the corpus callosum and the pyramidal tract meet.

\section{Conclusions}

We presented a method for fast calculation of higher-order tensor lines in both higher-order tensor representations as well as spherical harmonics representations of high angular resolution data. As the number of evaluations of the local data is small, the speed of our algorithm supersedes the speed of previous algorithms and is comparable to implementations of second-order tensors lines, where checks of the eigenvector orientation and direction corrections slow down the integration. We have shown that our implementation outperforms previous algorithms in both speed and precision. Especially in areas where maxima lie close together, our algorithm provides much higher resolution as sampling on a grid. Furthermore, it does not have problems with maxima artificially induced by the sampling structure as it is based on a continuous and smooth surface 

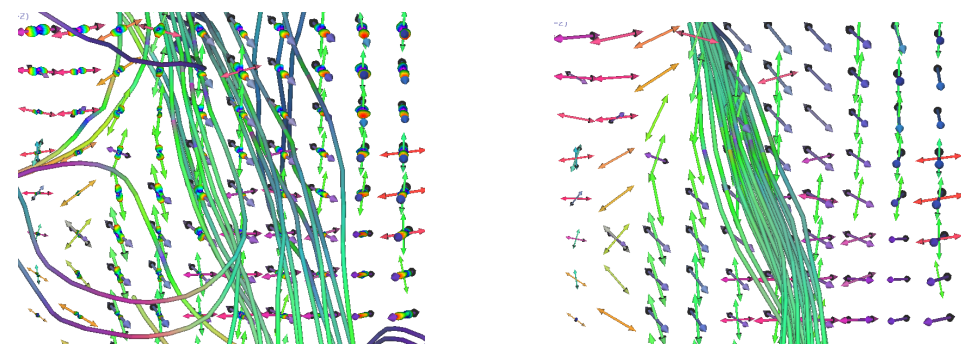

Fig. 6. Lines on second-order tensor field (left) and fourth-order data (right). The second order lines are not able to follow the right directions because of low fractional anisotropy, while higher-order lines find the dominant anterior-posterior fiber bundle in this area. The arrows indicate the grid-based directions at the sample points to get an approximate overview of the data, but are not used for the calculation.
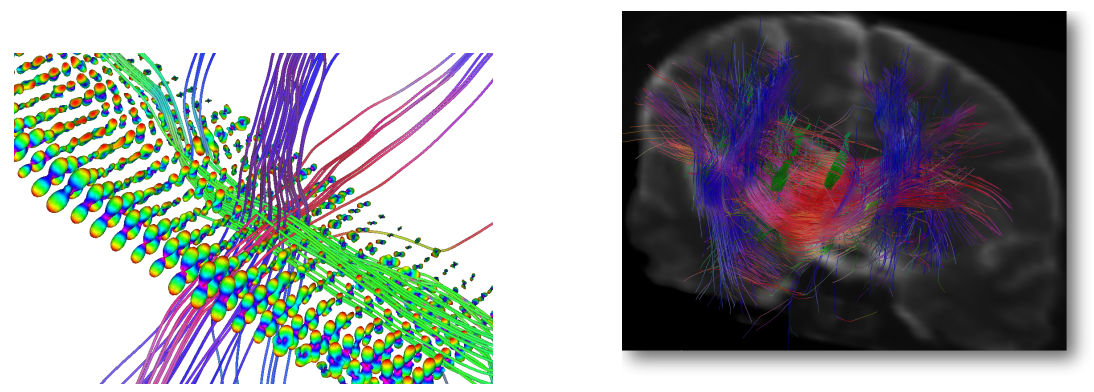

Fig. 7. Left: Crossing of three fibers out of a measured data set. Right: Fiber tracts in the same data set. The data has been acquired using a three-Tesla scanner at $b=2500$.

model. Thus, our main contribution is a fast and reliable algorithm that performs tracking of tensor lines in tensor fields of arbitrary order.

\section{Acknowledgments}

We thank Enrico Kaden and Davide Imperati for the fruitful discussion and the FAnToM-team, especially Alexander Wiebel at the University of Leipzig, Germany, Xavier Tricoche at Purdue University, West Lafayette, IN, USA, and Christoph Garth at the University of California, Davis, CA, USA for contributing code to the software framework used in this work.

\section{References}

1. Basser, P.J., LeBihan, D.: Fiber orientation mapping in an anisotropic medium with NMR diffusion spectroscopy. 11th Annual Meeting of the SMRM, Berlin (1992) 1221 
2. Basser, P., Mattiello, J., LeBihan, D.: Estimation of the effective self-diffusion tensor from the NMR spin echo. Journal of Magnetic Resonance 3 (1994) 247-254

3. Alexander, D.C.: An introduction to computational diffusion MRI: the diffusion tensor and beyond. In Weickert, J., Hagen, H., eds.: Visualization and Processing of Tensor Fields, Springer-Verlag Berlin Heidelberg (2006) 83-106

4. Frank, L.R.: Anisotropy in high angular resolution diffusion-weighted MRI. Magnetic Resonance in Medicine 45 (2001) 935-939

5. Frank, L.R.: Characterization of anisotropy in high angular resolution diffusionweighted MRI. Magnetic Resonance in Medicine 47 (2002) 1083-1099

6. Tuch, D.S., Reese, T.G., Wiegell, M.R., Makris, N., Belliveau, J.W., Wedeen, V.J.: High angular resolution diffusion imaging reveals intravoxel white matter. Magnetic Resonance in Medicine (2002) 577-582

7. Tuch, D.S.: Diffusion MRI of Complex Tissue Structure. PhD thesis, Massachusetts Institute of Technology (2002)

8. Alexander, D.C.: Persistent angular structure: new insights from diffusion magnetic resonance imaging data. Inverse Problems 19 (2003) 1031-1046

9. Özarslan, E., Mareci, T.H.: Generalized diffusion tensor imaging and analytical relationships between diffusion tensor imaging and high angular resolution diffusion imaging. Magnetic Resonance in Medicine 50 (2003) 955-965

10. Vilanova, A., Zhang, S., Kindlmann, G., Laidlaw, D.: An introduction to visualization of diffusion tensor imaging and its applications. In Weickert, J., Hagen, H., eds.: Visualization and Processing of Tensor Fields, Springer-Verlag Berlin Heidelberg (2006) 121-153

11. Blaas, J., Botha, C.P., Vos, F.M., Post, F.H.: Fast and reproducible fiber bundle selection in DTI visualization. In Silva, C.T., Gröller, E., Rushmeier, H., eds.: Proceedings of IEEE Visualization 2005, Los Alamitos, CA, USA, IEEE Computer Society, IEEE Computer Society Press (2005) 59-64

12. Weinstein, D.M., Kindlmann, G.L., Lundberg, E.C.: Tensorlines: Advectiondiffusion based propagation through diffusion tensor fields. In: Proceedings of IEEE Visualization '99, Los Alamitos, CA, IEEE Computer Society (1999) 249253

13. Hlawitschka, M., Scheuermann, G.: HOT-lines - tracking lines in higher order tensor fields. In Silva, C.T., Gröller, E., Rushmeier, H., eds.: Proceedings of IEEE Visualization 2005. (2005) 27-34

14. Descoteaux, M., Deriche, R., Lenglet, C.: Diffusion tensor sharpening improves white matter tractography. In: SPIE Medical Imaging, San Diego, California, USA (2007)

15. Tricoche, X., Scheuermann, G.: Topological methods for tensor visualization. In Hansen, C., Johnson, C.R., eds.: Visualization Handbook, Elsevier, Amsterdam (2004)

16. Abramowitz, M., Stegun, I.A.: Handbook of Mathematical Functions with Formulas, Graphs, and Mathematical Tables. ninth dover printing, tenth gpo printing edn. Dover, New York (1964)

17. Ivanic, J., Ruedenberg, K.: Rotation matrices for real spherical harmonics. Journal of Physical Chemistry 100 (1996) 6342-6347

18. Ivanic, J., Ruedenberg, K.: Corrections of rotation matrices for real spherical harmonics. Journal of Physical Chemistry A 102 (1999) 9099f

19. Descoteaux, M., Angelino, E., Fitzgibbons, S., Deriche, R.: Regularized, fast, and robust analytical q-ball imaging. Magnetic Resonance in Medicine (2007, to appear) 